\title{
Initial validations for pursuing irradiation using a gimbals tracking system.
}

\section{$\operatorname{AUTHOR}(\mathrm{S})$ :}

Takayama, Kenji; Mizowaki, Takashi; Kokubo, Masaki; Kawada, Noriyuki; Nakayama, Hiroshi; Narita, Yuichiro; Nagano, Kazuo; Kamino, Yuichiro; Hiraoka, Masahiro

\section{CITATION:}

Takayama, Kenji ...[et al]. Initial validations for pursuing irradiation using a gimbals tracking system.. Radiotherapy and oncology : journal of the European Society for Therapeutic Radiology and Oncology 2009, 93(1): 45-49

\section{ISSUE DATE:}

2009-10

URL:

http://hdl.handle.net/2433/91546

\section{RIGHT:}

c 2009 Elsevier Ireland Ltd. All rights reserved.; This is not the published version. Please cite only the published version.; この論文は出版社版で ありません。引用の際には出版社版をご確認ご利用ください。 


\section{Initial validations for pursuing irradiation using a gimbals tracking system}

\section{Authors:}

Kenji Takayama, M.D. ${ }^{* \dagger}$, Takashi Mizowaki, M.D., PH.D. ${ }^{*}$, Masaki Kokubo, M.D., PH.D. ${ }^{\dagger}$, Noriyuki Kawada, B.S. ${ }^{\dagger}$, Hiroshi Nakayama, B.S. ${ }^{\dagger}$ Yuichiro Narita, PH.D. ${ }^{*}$, Kazuo Nagano ${ }^{\dagger}$, Yuichiro Kamino, M.S. ${ }^{\ddagger}$, and Masahiro Hiraoka, M.D., PH.D. ${ }^{*}$

\footnotetext{
* Department of Radiation Oncology and Image Applied Therapy, Graduate School of Medicine, Kyoto University, Kyoto, JAPAN

$\dagger^{\dagger}$ Institute of Biomedical Research and Innovation, Kobe, JAPAN

\$ Mitsubishi Heavy Industries, Ltd., Tokyo, JAPAN
}

Corresponding author: Takashi Mizowaki

Department of Radiation Oncology and Image Applied Therapy, Graduate School of Medicine, Kyoto University

Address: 54 Kawahara-cho Shogoin Sakyo-ku Kyoto, 606-8507, JAPAN

$\mathrm{Tel}=+81-75-751-3419$, Fax $=+81-75-771-9749$

E-mail=mizo@kuhp.kyoto-u.ac.jp

Number of pages: 14 pages including this title page, summary, text, Conflict of Interest Statement, Acknowledgements, References, and Figure legends.

Number of tables and figures: 2 figures and no table 
Running head: Gimbals tracking irradiation

Keywords: Pursuing irradiation, gimbals tracking, organ motion, movable phantom, image-guided radiotherapy (IGRT) 


\section{Summary}

Our newly designed image-guided radiotherapy (IGRT) system enables the dynamic tracking irradiation with a gimbaled X-ray head and a dual on-board kilovolt imaging subsystem for real-time target localization. Examinations using a computer-controlled three-dimensionally movable phantom demonstrated that our gimbals tracking system significantly reduced motion blurring effects in the dose distribution compared to the non-tracking state. 


\section{Introduction}

Organ motion is an important issue during external-beam irradiation of extra-cranial lesions, particularly in the intra-thoracic and upper-abdominal regions, in which a tumor may move $1-2 \mathrm{~cm}$ as a result of respiration $[1,2]$. This motion results in blurred dose distributions and an enlarged beam penumbra at the radiation field edge [3, 4]. Therefore, sufficiently large safety margins are required to compensate for motion effects when using conventional techniques. Several approaches have been used to minimize motion effects, including respiratory inhibition [5], breath-hold [6, 7], respiratory gating [8-10], and tracking.

Real-time tracking irradiation is classified into two subcategories, according to the delivery scheme [2]. The first is intercepting irradiation, in which a therapeutic beam is gated to irradiate a tumor at a planned position by intercepting the tumor trajectory. This is in contrast to pursuing irradiation or dynamic tracking, which involves irradiating the target continuously as it moves through three-dimensional (3D) space. Pursuing irradiation provides higher delivery efficiency and greater comfort than gating and intercepting irradiation, in which the relative low duty cycle (typically 30-50\%) prolongs treatment time. In a robot-mounted linear accelerator (LINAC) system [11], pursuing irradiation is achieved through the use of a robotic arm, whereas dynamic multi-leaf collimator (DMLC)-based tracking utilizes a moving aperture [12]. Alternatively, a robotic couch moving in real-time in response to organ motion has been considered [13], although this approach may be problematic in terms of patient discomfort and potential danger.

The image-guided radiation therapy (IGRT) system described here, which was designed for precise initial setup, high throughput, and pursuing irradiation of moving 
targets, was developed by Mitsubishi Heavy Industries in collaboration with Kyoto University and the Institute of Biomedical Innovation and Research. The system involves a novel gimbaled X-ray head that directs a multi-leaf collimator (MLC)-shaped beam to a designated point in real-time. This paper describes a novel method for pursuing irradiation, termed "gimbals tracking", and provides data demonstrating its efficacy in reducing motion-induced marginal blurring.

\section{Materials and Methods}

\subsection{IGRT and the gimbals mechanism}

The concept and configuration of this IGRT system using a gimbaled X-ray head were previously introduced by Kamino et al. [14]. Briefly, a compact, lightweight, C-band 6-megavolt (MV) LINAC was mounted on a gimbaled X-ray head with a MLC, and the entire moving unit was installed on a ring-shaped gantry within a crescent-shaped cover. The X-ray head can rotate along the two orthogonal gimbals (pan and tilt rotations) up to $+/-2.4^{\circ}$, which swings the MV beam up to $+/-4.2 \mathrm{~cm}$ in each direction from the isocenter on the isocenter plane perpendicular to the beam. In the gimbals tracking mode, this mechanism enables the MV beam to track a target in real time. Two imaging units, each consisting of a kilovolt $(\mathrm{kV}) \mathrm{X}$-ray tube and a flat panel detector (FPD), were mounted on the gantry and provided real-time orthogonal serial radiographs. A gantry-mounted electronic portal imaging device (EPID) provided the information of the MV beam shape and position.

In this study, a prototype IGRT system was used. One of the differences from the commercial system was the MLC; the prototype used in this study had 40 pairs of 4-mm-thick leaves, which made a $16-\times 16-\mathrm{cm}$ field at the isocenter, whereas the 
commercial version had 30 pairs of 5-mm-thick leaves, which provide a $15-\times 15-\mathrm{cm}$ field.

\subsection{Movable phantom system}

A three-dimensionally movable phantom was developed to evaluate pursuing irradiation; the mechanical characteristics and accuracy of this system have been described previously [15]. The phantom system consisted of a drive unit, a computer control unit, and a spherical phantom (diameter, $19 \mathrm{~cm}$ ). Radiographic film was inserted between the hemispheres of the phantom, and a copper plate was attached to the inside of the upper hemisphere to minimize film exposure. A pin-hole with a diameter of $2 \mathrm{~mm}$ at the center of the plate was used as a fiducial marker. The drive unit consisted of three linear stages designed to move the phantom according to the three-dimensional (3D) trajectory and velocity specified by the control unit.

\subsection{Film irradiation}

Irradiation tests using a prototype of our IGRT system were performed to evaluate the efficacy of gimbals tracking in reducing dose blurring. A 6-MV beam was used to irradiate an $8-\times 8$-mm field of film in the phantom under the following conditions:

(a) Stationary state: stationary phantom with a stationary X-ray head;

(b) Non-pursuing state: phantom in motion with a stationary X-ray head; and

(c) Pursuing irradiation: phantom in motion with gimbals tracking enabled.

The phantom moved in the horizontal plane, parallel to the film and perpendicular to the MV beam. The following motion patterns were tested:

(1) linear reciprocal motion of a triangular wave (stroke, $20 \mathrm{~mm}$; velocity, $10 \mathrm{~mm} / \mathrm{s}$ ); 
(2) circular motion on the horizontal plane (radius, $10 \mathrm{~mm}$; tangential velocity, $5 \mathrm{~mm} / \mathrm{s}$ ); and

(3) linear reciprocal motion of a respiration-like wave (stroke, $20 \mathrm{~mm}$ ).

During pursuing irradiation, the frame rate for real-time imaging was 7.5 frames/s, and an original predictive protocol based on a linear autoregressive model was applied to compensate for the mechanical and image processing lag of the tracking system. The Levinson-Durbin recursion algorithm was used to determine the coefficients of the model at a high speed [16]. This protocol allows prediction of the target position based on the past time series data and provides the gimbal control unit with positional information within milliseconds after obtaining the current position.

The irradiated film was developed, and its optical density was evaluated using a film analyzer. To mimic the clinical setting, motion effects in a 48- $\times 48$-mm field were also examined, using a respiratory-like wave, which was created from measured data for human abdominal wall motion.

\section{Results}

In every motion pattern, pursuing radiation using the method described here significantly reduced motion effects (i.e., blurring). Figure 1 shows the two-dimensional (2D) dose distributions and line dose profiles for an $8-\times 8-\mathrm{mm}$ field moving in a triangular wave or a circular motion. During linear reciprocal motion (Fig. 1[1]), the 2D dose distribution for the phantom showed significant marginal blurring, reflecting the motion probability density function (PDF). Pursuing irradiation dramatically reduced blurring and produced a dose profile slope similar to that of the stationary state $(<\sim 1 \mathrm{~mm})$. During circular motion (Fig. 1[2]), a faint circular dose distribution was obtained; by 
contrast, pursuing irradiation produced a square dose distribution, similar to that of the stationary state, with only slight marginal blurring in all directions.

Figure 2 demonstrates the efficacy of pursuing irradiation while irradiating a $48-\times$ 48-mm field in respiratory-like motion. The stationary X-ray head produced significant blurring while the phantom was in motion. The high-dose area, defined as the distance between $95 \%$ dose points in the left and right slopes, decreased to approximately $70 \%$ $(26.4 / 38.1)$ of the stationary state. The slope of the low-dose area, defined as the distance between 20 and $80 \%$ dose points, declined to approximately five-fold that of the stationary state. During pursuing irradiation, blurring was so slight that the high-dose area was equivalent to the stationary state and the slope was only 1.5 -fold that of the stationary state.

\section{Discussion}

The data presented here demonstrate the utility of gimbals tracking as a new method for pursuing irradiation. Gimbals tracking has three primary advantages. First, one-degree-ordered small-angle rotations of the gimbals provide quick and accurate beam adaptation to designated positions of a mobile target. Second, the mechanism is relatively simple and thus minimizes mechanical load. Finally, our system is safer than systems involving a robotic arm because the moving unit is covered.

However, we acknowledge that the beam path in the gimbals tracking system varies from the planned beam to some extent. This is one of the differences from the robot-mounted LINAC system, in which the beam path changes in parallel to the original path during beam tracking. Variation in the beam path is greatest when a target on the isocenter moves on a plane perpendicular to the beam. When the gimbaled head swings 
its maximum rotation by as much as $2.4^{\circ}$ along both the pan and tilt axes of the gimbals, the target can be located $5.9 \mathrm{~cm}$ from the isocenter on the plane, and the distance between the target and radiation source is $100.2 \mathrm{~cm}$. This discrepancy in distance compared to 100 cm means an approximate $1 \%$ dose change when calculated based on the percent depth dose (PDD) of the beam. Therefore, the effect of the beam path variation due to limited rotation of the gimbals appears to be reasonably small in terms of the dose. However, it would be better to simulate the impact of the described effects and to confirm whether possible dose differences are within clinically acceptable limits using a treatment planning system. Therefore, we plan to develop a treatment planning program for our system that will allow us to simulate and evaluate the dose distribution of tracking irradiation with a gimbal-mounted head.

In stereotactic body radiotherapy, gimbals tracking provides higher delivery efficiency than either gating or robotic tracking, and in comparison with robotic tracking it produces a more homogeneous in-field dose distribution, without cold spots. This is because, as in DMLC tracking, gimbals tracking uses ten or fewer flattened beams that are MLC-shaped to include the whole target in the beam's eye view. By contrast, robotic tracking is more time-consuming because several tens to 100 narrow conical beams are used, creating greater inhomogeneity in the dose distribution profiles compared with those of conventional MLC. Moreover, tracking error may occur independently in each beam, resulting in unpredictable hot and cold spots in the target.

Strictly speaking, the movement of a target in the human body involves both positional change and deformation. However, the deformation is relatively small for a small solitary tumor without lymph node metastasis, which is the most appropriate candidate for pursuing irradiation in general, rather than a large tumor, which may have a 
complex shape, subclinical extension, and lymph node metastasis. However, if deformation could be detected by an imaging system, such as a four-dimensional (4D) computed tomography (CT) scanner, a 4D treatment planning system considering these movements would help to cope with this issue. We have been developing an original 4D planning system at Kyoto University. If these technologies were available, gimbals tracking could be achieved by adjusting the irradiation fields to cover the entire target, including deformation. This is an advantage over a robot-mounted LINAC system, which would not easily compensate for deformation.

In terms of real-time imaging, our system uses a gantry-mounted stereo $\mathrm{kV}$ X-ray imaging system to detect real-time 3D positional information for a mobile target. This technique is capable of directly tracking tumors based on the density difference between the tumor and normal lung tissue, provided that the tumor is well defined with a high-contrast edge. Several variations of our tracking system would be possible in a clinical setting, such as direct tracking, external surrogates, and internal surrogates (fiducial markers, diaphragms, etc.). Further research is required to develop prediction techniques and correlation models for the surrogate signal versus internal tumor motion. The EPID allows visualization of the radiation field aperture and the tumor/internal surrogate, and thus may play an important role in verifying MV beam allocation.

Regarding the validation data presented in this study, our results showed that a 48$\times 48-\mathrm{mm}$ field produced a $38-\mathrm{mm}$ high-dose area exposed to more than $95 \%$ of the dose in the stationary state (See Fig. 2). However, motion blurring in the non-pursuing state decreased the width of the high-dose area to $26.4 \mathrm{~mm}$, which theoretically means that the field size in the target motion direction should have been enlarged to about $60 \mathrm{~mm}$ to create a similarly sized high-dose area. With pursuing irradiation, even if the slight 
marginal blurring is considered, a 50- $\mathrm{mm}$ field was large enough to create the same high-dose area. The slight blurring demonstrated in this study arose for several reasons, such as delays in image processing and communication, prediction error, and a mechanical response time lag. In fact, the response delay of the gimbals during the actual test was a maximum $0.4-0.6 \mathrm{~mm}$ to sinusoidal motion, as mentioned in Kamino et al. [14]. Further investigation of these factors would improve the tracking accuracy of our system. Another solution to improve prediction accuracy may be to increase the sampling rate or imaging frame rate. However, the sampling rate is limited by processing time, and the rate we used in this study ( 7.5 frames/s) is the maximum possible using the system described here. Excessively high imaging frame rates are not feasible in clinical practice because of exposure to the imaging dose.

Although a few potential problems remain to be resolved, our data indicate that this gimbals tracking system is well balanced and potentially ideal for realizing pursuing irradiation.

\section{Conclusion}

A movable phantom was used to examine the basic capabilities of a novel, gimbal-mounted IGRT system. Pursuing irradiation with this system significantly reduced motion-induced marginal blurring. Further research is underway to refine this technique for clinical use. 


\section{Conflict of Interest Statement:}

Kenji Takayama, Noriyuki Kawada, Hiroshi Nakayama, and Yuichiro Kamino are employees of Mitsubishi Heavy Industries and have been developing the image-guided radiation therapy (IGRT) system described here. Takashi Mizowaki, Masaki Kokubo, and Masahiro Hiraoka have a consultancy agreement with Mitsubishi Heavy Industries.

\section{Acknowledgments:}

This work was partially supported by Grants-in-Aid for scientific research from the Ministry of Education, Culture, Sports, Science, and Technology (20229009) of Japan; Grants-in-Aid for Scientific Research on Priority Areas Cancer from the Ministry of Education, Culture, Sports, Science, and Technology (17016036); the Association for Nuclear Technology in Medicine; and the New Energy and Industrial Technology Development Organization (NEDO) in Japan. These study sponsors played no role in the study design, collection, analysis and interpretation of data, writing of this manuscript, or the decision to submit this manuscript for publication.

The first author gratefully acknowledges Ayuko Yamaguchi for her editorial assistance in English, and her private and spiritual support as his affectionate wife.

This work was partially presented at the $47^{\text {th }}$ Annual Meeting of American Society for Therapeutic Radiology and Oncology, October 2005, Denver, CO. 


\section{References}

[1] Keall PJ, Mageras GS, Balter JM, et al. The management of respiratory motion in radiation oncology report of AAPM Task Group 76. Med Phys 2006;33:3874-3900.

[2] Shirato H, Suzuki K, Sharp GC, et al. Speed and amplitude of lung tumor motion precisely detected in four-dimensional setup and in real-time tumor-tracking radiotherapy. Int J Radiat Oncol Biol Phys 2006;64:1229-1236.

[3] Bortfeld T, Jiang SB, Rietzel E. Effects of motion on the total dose distribution. Semin Radiat Oncol 2004;14:41-51.

[4] McCarter SD, Beckham WA. Evaluation of the validity of a convolution method for incorporating tumour movement and set-up variations into the radiotherapy treatment planning system. Phys Med Biol 2000;45:923-931.

[5] Negoro Y, Nagata Y, Aoki T, et al. The effectiveness of an immobilization device in conformal radiotherapy for lung tumor: reduction of respiratory tumor movement and evaluation of the daily setup accuracy. Int J Radiat Oncol Biol Phys 2001;50:889-898.

[6] Onishi H, Kuriyama K, Komiyama T, et al. A new irradiation system for lung cancer combining linear accelerator, computed tomography, patient self-breath-holding, and patient-directed beam-control without respiratory monitoring devices. Int $\mathbf{J}$ Radiat Oncol Biol Phys 2003;56:14-20.

[7] Wong JW, Sharpe MB, Jaffray DA, et al. The use of active breathing control (ABC) to reduce margin for breathing motion. Int J Radiat Oncol Biol Phys 1999;44:911-919.

[8] Ohara K, Okumura T, Akisada M, et al. Irradiation synchronized with respiration 
gate. Int J Radiat Oncol Biol Phys 1989;17:853-857.

[9] Kubo HD, Hill BC. Respiration gated radiotherapy treatment: a technical study. Phys Med Biol 1996;41:83-91.

[10] Wurm RE, Gum F, Erbel S, et al. Image guided respiratory gated hypofractionated Stereotactic Body Radiation Therapy (H-SBRT) for liver and lung tumors: Initial experience. Acta Oncol 2006;45:881-889.

[11] Murphy MJ. Tracking moving organs in real time. Semin Radiat Oncol 2004;14:91-100.

[12] Keall PJ, Joshi S, Vedam SS, et al. Four-dimensional radiotherapy planning for DMLC-based respiratory motion tracking. Med Phys 2005;32:942-951.

[13] D'Souza WD, Naqvi SA, Yu CX. Real-time intra-fraction-motion tracking using the treatment couch: a feasibility study. Phys Med Biol 2005;50:4021-4033.

[14] Kamino Y, Takayama K, Kokubo M, et al. Development of a four-dimensional image-guided radiotherapy system with a gimbaled X-ray head. Int J Radiat Oncol Biol Phys 2006;66:271-278.

[15] Nakayama H, Mizowaki T, Narita Y, et al. Development of a three-dimensionally movable phantom system for dosimetric verifications. Med Phys 2008;35:1643-1650.

[16] Durbin J. The fitting of time series models. Rev Inst Int Sta 1960; 28:233-243. 


\section{Figure legends}

Fig. 1 Film irradiation experiments using an $8-\times 8$-mm field. Two-dimensional dose distributions and line dose profiles are shown for each motion pattern. After background compensation, the profiles were normalized for integral dose.

Fig. 2 Film irradiation experiment using a $48-\times 48-\mathrm{mm}$ field. The line dose profiles were normalized at the field center. The effect of pursuing irradiation on motion-induced marginal blurring is visually and numerically demonstrated. 
Motion pattern 2D dose distribution Line dose profile

[1] linear reciprocal motion of a triangular wave
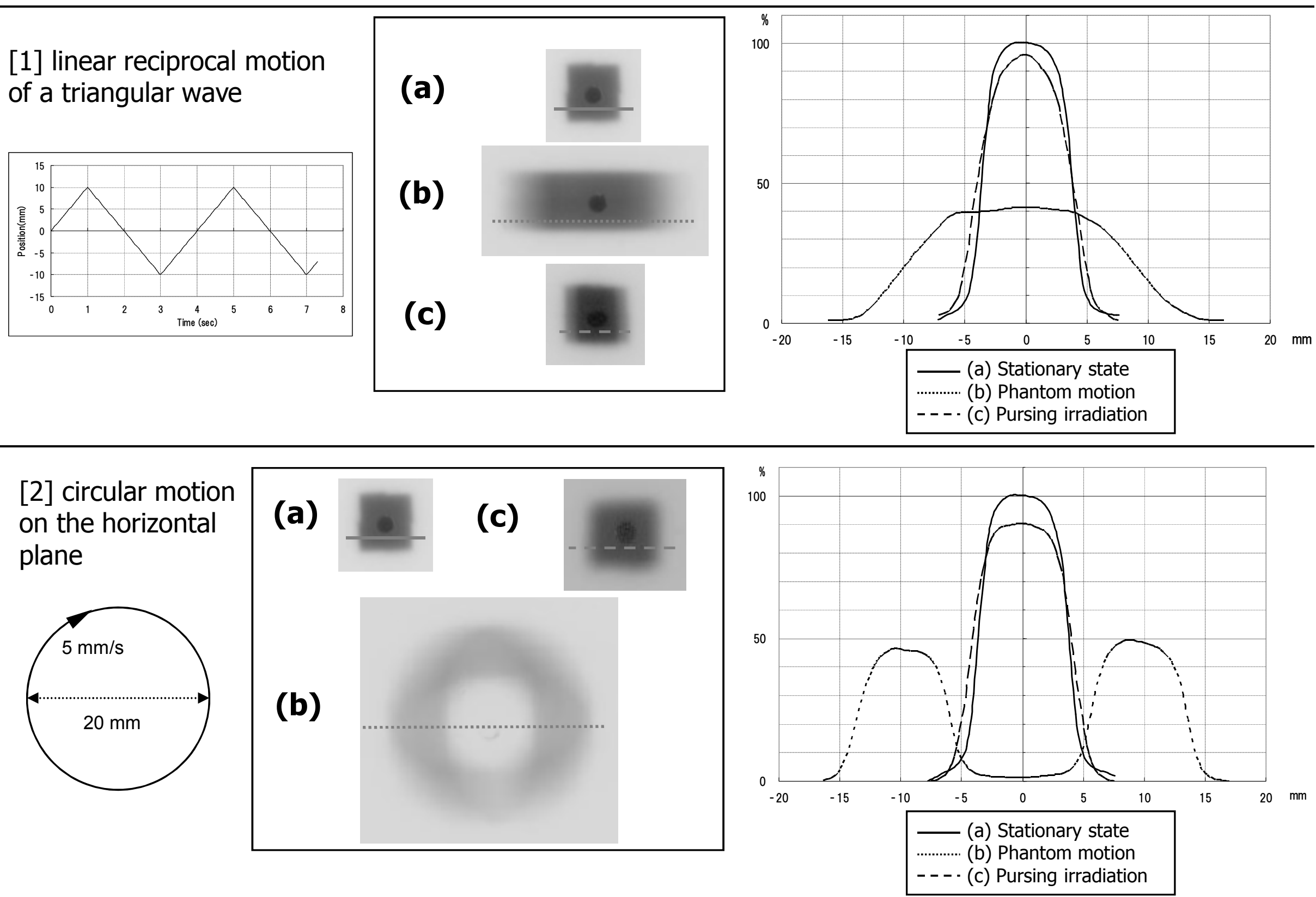

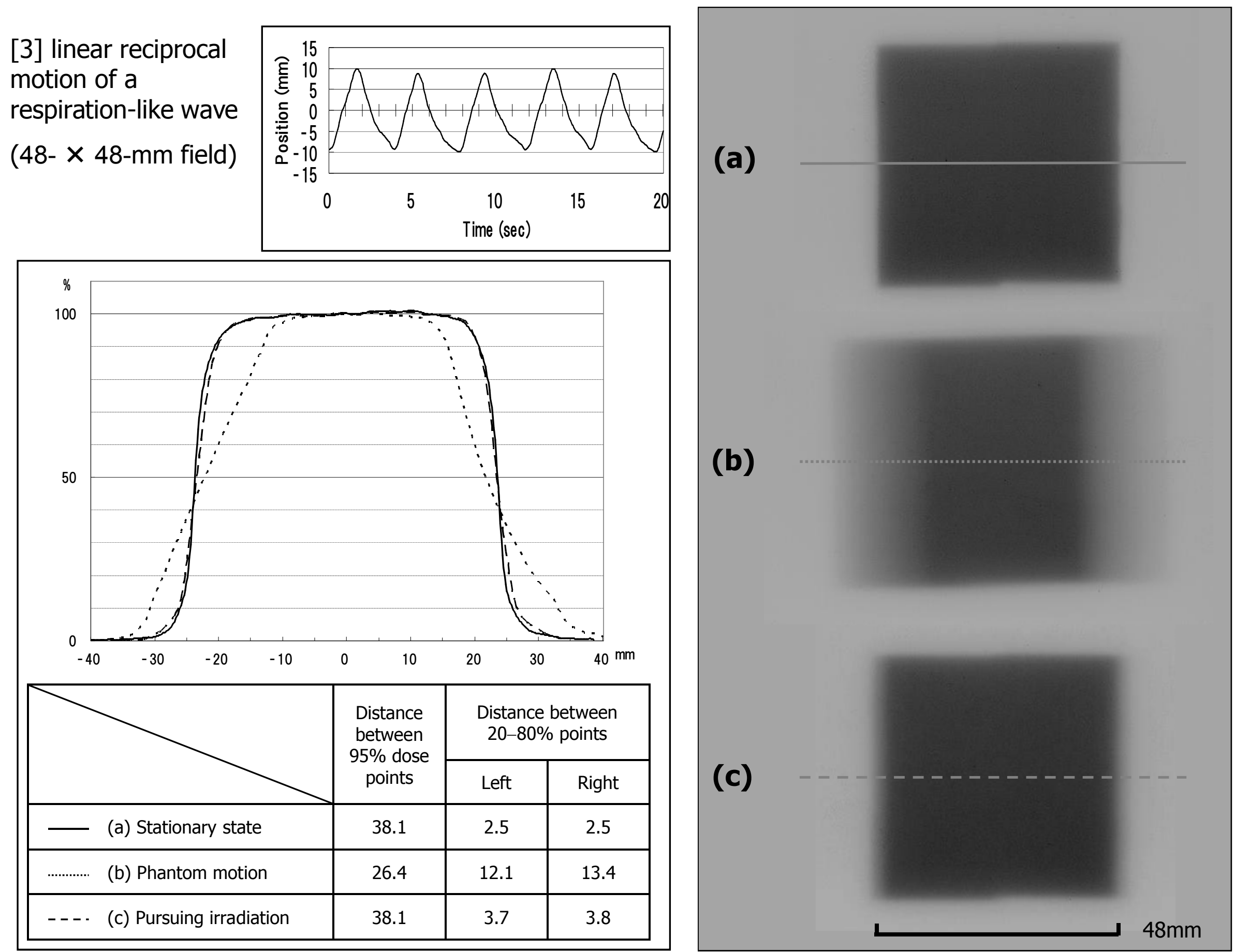